\title{
Programas de especialización de medicina interna en el Caribe colombiano Una necesidad latente
}

\section{Programs of specialization in internal medicine in the colombian Caribbean \\ A latent necessity}

Rubén Camargo, Jorge Bilbao, Rusvelt Franklin Vargas

- BarRanquilla (Colombia)

\section{Introducción}

La medicina interna, comprendida como una especialidad clínica, enfocada en el adulto, se caracteriza por su posición de "integralidad en el enfoque" y de evaluación activa en todos los aspectos relevantes para la salud del individuo teniendo en cuenta los determinantes del proceso salud-enfermedad, mediante un conocimiento científico profundo de la fisiopatología del ser humano. Así mismo, tiene en cuenta los aspectos diagnósticos y terapéuticos, a través del empleo de los recursos médicos disponibles.

El especialista en medicina interna debe tener una formación sólida a nivel profesional, que le permita valorar y transformar el estado de salud del adulto, siempre con un enfoque biopsicosocial.

La medicina interna es una rama del saber que estudia y trata las enfermedades del adulto que no suelen ser objeto de tratamiento quirúrgico. Es un recurso humano capacitado y calificado en atender patologías crónicas y patologías agudas de enfermedades neurológicas, cardiológicas, neumológicas, gastrointestinales. Haciendo que el internista sea una persona capacitada para atender integralmente al paciente en su medio interno.

\section{La medicina interna en el contexto internacional}

A nivel internacional la demanda de internistas es fuerte y es la posición más solicitada en los hospitales, ya que posee un rol integrador inherente, que considera al paciente desde una perspectiva global, acompañándolo como médico tratante o consultor a lo largo de su vida adulta (1).

Una de las principales razones por las que se aumenta cada día la demanda de médicos internistas se debe a que, conforme se incrementa la esperanza de vida la necesidad de los médicos se aumenta, debido a que todo un conjunto de problemas médicos se pueden presentar a medida que transcurren los años vividos por las personas, y precisamente la medicina interna se ocupa de estos elementos.

De la misma forma, dado que vivimos en un mundo cada vez más globalizado, las fronteras para el ejercicio profesional tienden a disminuirse. Por ejemplo, en España la internacionalización de la profesión médica es una realidad. Según Barber y González (2), en promedio del año 2004-2009 se homologaron 3280 títulos de licenciatura en medicina cada año de países ajenos a la Unión Europea, sobre todo de Latinoamérica.

Las migraciones de los médicos han recibido en los últimos años gran atención por parte de organismos internacionales, como la OMS, en el informe sobre la salud en el mundo 2006, y la OCDE, que tiene un programa específico sobre el tema (3). Según dicho informe entre 1990 y 2005, el número anual de nuevos licenciados en medicina tuvo una gran disminución en muchos países, particularmente en Francia, Alemania, Italia, Japón, España y Suiza, y alerta sobre la tendencia deficitaria global que se palia con movimientos migratorios internacionales.
Dr. Rubén Camargo Rubio: Especialista en Medicina Interna y Medicina Intensiva. Presidente Asociación Colombiana de Medicina Crítica y Cuidado Intensivo. Coordinador Especialización en Medicina Interna Fundación Universitaria San Martín, Director Científico Clínica General del Norte; Dr. Jorge Bilbao Ramírez: Sociólogo, Magíster en Salud Pública y en Educación, Especialista en Salud Ocupacional, Candidato a Doctorado en Educación. Jefe Área de Formación Investigativa Fundación Universitaria San Martín; Dr. Rusvelt Franklin Vargas Moranth: Epidemiólogo, Docente Fundación Universitaria San Martín. Candidato a Magíster en Salud Pública. Barranquilla (Colombia).

Correspondencia. Dr. Rusvelt Franklin Vargas Moranth. Barranquilla (Colombia). E-mail: rfvargas1@hotmail.com Recibido: 11/XI/2012 Aceptado: 27/VI/2013 
En España, el número de médicos especialistas en activo asciende a 141579 con una tasa de 319 por cada 100000 habitantes, y un défict de $2 \%$ en 2008 , proyectado a $14 \%$ en 2025 (2).

En Estados Unidos el estándar de planificación es un médico por 2000 habitantes. Los estándares de necesidades de especialistas que se manejan en este país son la base para muchos otros alrededor del mundo (4) (Tabla 1).

En países en los cuales el papel del internista es más resolutivo, y constituye en último eslabón de consulta, estos valores difieren un poco. Es así como en Canadá (5) se maneja un estándar de 11 médicos internistas por cada 100000 habitantes, y en el Reino Unido (6) este valor es de 1,2 por cada 100000 habitantes.

Es importante así mismo, el papel que realizan los avances tecnológicos en los cambios de necesidad de especialistas, en la medida en que la necesidad se define como la capacidad del especialista, empleando el conocimiento y los instrumentos diagnósticos y terapéuticos al día, para mejorar la salud de las personas y poblaciones. Según un estudio publicado por la Health Rersources and Services Administration (7), para EE.UU., en el escenario base de crecimiento económico moderado, en el 2020 se requerirán $22 \%$ más médicos que en 2005.

\section{La medicina interna en el contexto nacional}

Dentro del sistema de salud de nuestro país, el especialista en medicina interna es tomado como médico de referencia en el segundo y tercer nivel de atención, y en la práctica privada, actúa como médico consultor.

Existen dos elementos que plantean un manejo integral y a la vez preventivo de las patologías propias del abordaje de la medicina interna:

1. Un aumento de la expectativa de vida la población. Según el Departamento Administrativo Nacional de Estadísticas (8), la esperanza de vida al nacer para el periodo 2005-2010 fue de 74 años, y para el Departamento del Atlántico este valor es superior al promedio nacional: 74.8. Si a esto se le añade que las proyecciones muestran

Tabla 1. Estándares de necesidad de médicos internistas y de especialidades derivadas, por cada 100000 habitantes.

\begin{tabular}{|ccc|}
\hline Edad & Medicina interna & $\begin{array}{c}\text { Otras derivadas } \\
\text { de la Medicina Interna }\end{array}$ \\
\hline $\mathbf{1 8 - 2 4}$ & 43 & 15 \\
$\mathbf{2 5 - 4 4}$ & 59 & 23 \\
$\mathbf{4 5 - 6 4}$ & 89 & 41 \\
$\mathbf{6 5 - 7 4}$ & 175 & 97 \\
$\mathbf{7 5 +}$ & 270 & 130 \\
\hline Total & 95 & 33 \\
\hline Fuente: U.S. Department of Health and Human Services Health Resources and Services \\
Administration Bureau of Health Professions Physician Supply and Demand: Projec- \\
tions. to 2020. Octubre 2006. \\
\hline
\end{tabular}

que este indicador se incrementará (Figura 1), esto indica que habrá una mayor población objetivo del estudio de la medicina interna.

2. El desarrollo tecnológico en la actualidad es cada vez más creciente y, junto con el incremento de la expectativa de vida de la población, ponen sobre el tapete plantean la necesidad de llevar a cabo un manejo integral y preventivo de las enfermedades tanto crónicas como degenerativas propias de la especialidad de la medicina interna, como es el caso de las enfermedades cardiovasculares, las cuales constituyen la primera causa de morbimortalidad en el mundo occidental y la segunda causa de muerte en Colombia. El infarto agudo de miocardio (IAM) es la patología más frecuente dentro de este grupo de enfermedades.

Este hecho ha coincidido con una serie de cambios en los hábitos que acompañan el proceso socioeconómico del desarrollo y que se relacionan directamente con la transición de una sociedad tradicional rural a una sociedad en la que la mayoría de la población vive en las grandes urbes y adopta los hábitos ligados a la sociedad industrial y consumista.

De acuerdo al documento "Situación de Salud en Colombia - Indicadores Básicos 2001", publicado por el Ministerio de Salud y la Organización Mundial de la Salud en Colombia, la mortalidad por enfermedades cardiovasculares en Colombia en el año 1998 es de 113.4 por 100000 habitantes (9). Estos valores no han mostrado mayor variación en los últimos años.

Según la Dirección Seccional de Salud de Antioquia, las enfermedades isquémicas en 1999 tenían una tasa de mortalidad de 52.9/100000 habitantes y ha ido aumentando a partir de ese año, hasta llegar al 2004, a un total de 3857 muertes (hombres 2142 y mujeres 1715), con una tasa por 100000 habitantes de 67.8 (hombres 78.1 y mujeres 58.3). Se

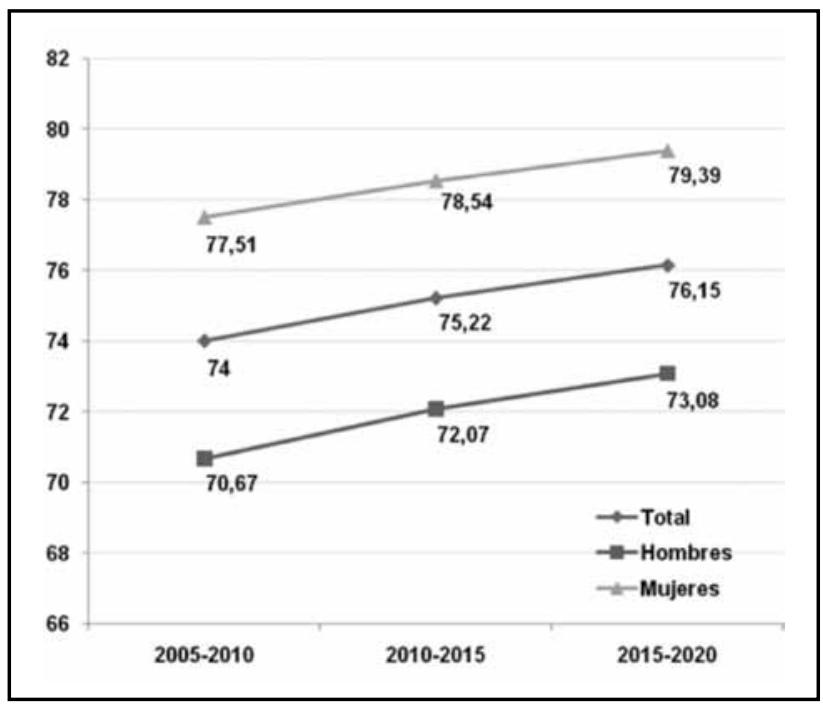

Figura 1. Esperanza de vida al nacer para la población Colombiana, 2005-2020. Fuente DANE 2005 
mantuvo casi estable en el 2005 con una tasa de mortalidad de 61.5/100000 habitantes; convirtiéndose en la primera causa de mortalidad en este departamento (10).

En el 2004, la cuarta causa de muerte en el departamento del Atlántico, correspondió a enfermedades cardiovasculares (33.89\%) y dentro de éstas a la enfermedad isquémica le corresponde un $19.23 \%$. Así mismo se encontró que en el grupo de edad de 45-59 años, en la misma fecha, la enfermedad isquémica fue la primera causa de mortalidad con 20.24\% que corresponde a 50 casos de 247 muertes en ese grupo de edad. En las personas de 60 años o más, la enfermedad isquémica también fue la primera causa de muerte con $26.94 \%$, que corresponde a 302 casos de 1121 muertes en este grupo etáreo (11).

Según el Céndex (12) la mayoría de la carga de enfermedad según AVISAS, corresponde a problemas definidos como pertenecientes al grupo II o grupo de enfermedades crónicas, no transmisibles ( $76 \%), 81 \%$ en mujeres y $72 \%$ en hombres. El grupo I constituido por las condiciones transmisibles, maternas, perinatales y de la nutrición tiene un peso de $15 \%$ en la carga global, $14 \%$ en hombres y $16 \%$ en mujeres; el grupo III o de lesiones, es responsable de $9 \%$ de la carga de enfermedad, correspondiendo 14\% en hombres y sólo $3 \%$ en mujeres. En hombres el peso del grupo III alcanza 14\% del total mientras que en mujeres llega sólo a 3\%.

Según un estudio realizado por Osorio (13), en nuestro país el número de internistas y subespecialistas en áreas de la medicina representa $18.86 \%$ del total de especialistas registrados, para una tasa de 0.949 internistas por 10000 habitantes.

\section{La medicina interna en el contexto local}

El campo de acción de la medicina interna en el contexto local, resulta de gran amplitud, ya que esta rama de las ciencias médicas se enfoca tanto en el diagnóstico y tratamiento de las enfermedades infecciosas, como en las crónicas y ambas son de gran impacto en términos de morbilidad y mortalidad en el departamento del Atlántico.

Por otro lado, la oferta de servicios da cuenta de un terreno que amerita la participación de profesionales en este campo. Según Orozco (14), con datos del año 2008 en el departamento del Atlántico, había 555 IPS en los diferentes grados de complejidad, de las cuales 78 son entidades públicas $(14 \%)$ y 477 son de carácter privado, lo cual podría considerarse como una oferta amplia para el tamaño total de la población, aunque la mayoría se encuentran concentradas en la ciudad de Barranquilla y en la ciudad de Soledad.

De los servicios hospitalarios ofertados por la Red Pública del departamento, $27.27 \%$ corresponde a servicios de hospitalización general para adultos, y dentro de los servicios hospitalarios ofertados, el departamento cuenta con una amplia disponibilidad de camas, con un total de 2781 camas, distribuidas: 39.19\% en IPS de carácter público es decir 1090 camas del total de la oferta departamental, con $60.81 \%$ en IPS privadas, es decir 1691 camas en la red privada.
Así mismo, de los 947 servicios de medicina especializada ofertados por profesionales independientes en el departamento solamente $6.86 \%$ corresponden a medicina interna

\section{Estado de la educación en medicina interna}

En la región Costa Caribe se encuentran programas de medicina interna en los departamentos de: Atlántico, Bolívar, Córdoba, Magdalena. En la ciudad de Barranquilla se encuentran programas, en la Universidad del Norte, Universidad Libre y Metropolitana, con un promedio de egresados de 25 especialistas en medicina interna anualmente, cifra que se ha incrementado desde que se iniciaron los posgrados en la ciudad de Barranquilla en 1999.

En este sentido los recursos humanos en salud se generan en el mercado educativo, adquiriendo ciertas habilidades específicas y pasan a desempeñar sus actividades profesionales ingresando al mercado de trabajo, en el cual deciden cuál magnitud de su tiempo y esfuerzo ofrecen, de acuerdo con el juego de preferencias entre el trabajo y otras actividades diferentes a éste y, de la misma manera, teniendo en cuenta los ingresos laborales que pueden obtener con la puesta en práctica de sus conocimientos profesionales y los beneficios o satisfacciones derivados de actividades alternativas que pueden ejercer.

\section{Impacto y futuro de la medicina interna}

La medicina interna ha ido evolucionando, hasta el punto de convertirse en una disciplina que abarca los aspectos más importantes del cuidado integral del adulto, comenzando por la prevención, pasando por el diagnóstico hasta llegar al tratamiento de patologías, tanto agudas como crónicas con un manejo del paciente tanto en la consulta externa como a nivel hospitalario.

La complejidad de la asistencia a nivel hospitalario en el contexto mundial actual, el cual se tecnifica cada vez más, implica que los pacientes tengan la necesidad de disponer de un médico que ofrezca, no solo su atención integral, su defensa y consejo profesionalizado, sino un rol que debe ser protagónico.

Por otro lado, las pirámides poblacionales actuales por especialidad, son un factor decisivo en la evolución de la tendencia de recursos humanos médicos. En las especialidades tradicionales, como el caso de la medicina interna, con una fuerza de trabajo en los intervalos etarios mayores, se determinará una dinámica de reposición muy diferente a aquellas otras especialidades en las que los recursos humanos son significativamente más jóvenes.

\section{Referencias}

1. Varela Nacor. Ejercicio de la Medicina Interna en América Latina. Rol de médico internista. Rev Med Chile 2002; 130: 1.

2. Barber P. González B. Oferta y necesidad de médicos especialistas en España (2008-2025). Universidad de Las Palmas de Gran Canaria. Diciembre 2008.

3. Dumont JC, Zurn P., Church J., Le Thi C. International Mobility of Health Professionals and Health Workforce Management in Canada: Myths and Realities. OECD. Health working paper 2008; Octubre (40).

4. U.S. Department of Health and Human Services Health Resources and 
Services. Administration Bureau of Health Professions Physician Supply and Demand: Projections. to 2020. Octubre 2006. Accesible en http://bhpr.hrsa.gov/ healthworkforce/reports/physiciansupplydemand/growthandaging.htm. Fecha de acceso: Marzo de 2011

5. Lorne Verhulst CBF, Mike M. To Count Heads or To Count Services? Comparing Population-to-Physician Methods with Utilization-Based Methods for Physician Workforce Planning: A Case Study in a Remote Rural Administrative Region of British Columbia. Healthcare Policy / Politiques de SantÈ 2007; 2(4).

6. The Federation of The Royal Colleges of Physicians of The United Kingdom. Consultant Physicians in the UK, 2007. Data and Commentary.

7. Health Resources and Services Administration (HRSA). Tomado de: http:// bhpr.hrsa.gov/healthworkforce/reports/physiciansupplydemand/growthandaging. htm. Fecha de acceso: marzo de 2011.

8. DANE. El Censo 2005 dejar ver una nueva realidad demográfica. Comunicado de Prensa. Tomado de: http://www.dane.gov.co/files/BoletinProyecciones.pdf Fecha de acceso: febrero de 2011

9. Asociación Panamericana De Gerencia De Servicios De Salud. Situación De
Salud en Colombia: Indicadores Básicos 2.001 http://www.gerenciasalud.com/ art42.htm. Accesado: 23 de abril del 2008.

10. Dirección Seccional De Salud De Antioquia. Mortalidad por infarto agudo de miocardio por municipios y regiones Departamento de Antioquia 1994-2005 Disponible en Internet: http://www.dssa.gov.co/html/mortalidad.htm. Accesado: noviembre de 2008 .

11. Navarro E, Tuesca R, Barceló M, Vargas R, Sierra C. Análisis de la situación de salud: Departamento del Atlántico 2004-2005. Barranquilla: Ediciones Uninorte 2006.

12. Cendex. Carga de enfermedad Colombia 2005: resultados alcanzados. Documento Técnico ASS/1502-08. Bogotá, Octubre 2008.

13. Osorio J. Oferta y demanda de profesionales en el sector salud, un problema de mercado. CES Medicina 2004; 18: 69-75.

14. Orozco Africano, J.M. Estudio de factibilidad para la creación de una IPS privada de tercer nivel de atención en el distrito de Barranquilla, <="' i="'>Edición electrónica gratuita. Texto completo en www.eumed.net/libros/2008b/385/ Fecha de acceso: marzo de 2011. 